AperTO - Archivio Istituzionale Open Access dell'Università di Torino

\title{
Cross-Cultural Validation of the Rorschach Developmental Index
}

\section{This is the author's manuscript}

Original Citation:

Availability:

This version is available http://hdl.handle.net/2318/158704

since 2016-06-30T17:23:53Z

Published version:

DOI:10.1080/00223891.2014.960927

Terms of use:

Open Access

Anyone can freely access the full text of works made available as "Open Access". Works made available under a Creative Commons license can be used according to the terms and conditions of said license. Use of all other works requires consent of the right holder (author or publisher) if not exempted from copyright protection by the applicable law. 
This is the author's final version of the contribution published as:

Luciano Giromini;Donald J. Viglione;Emanuela Brusadelli;Margherita Lang;Jennifer B. Reese;Alessandro Zennaro. Cross-Cultural Validation of the Rorschach Developmental Index. JOURNAL OF PERSONALITY ASSESSMENT. 97 (4) pp: 348-353.

DOI: $10.1080 / 00223891.2014 .960927$

The publisher's version is available at:

http://www.tandfonline.com/doi/full/10.1080/00223891.2014.960927

When citing, please refer to the published version.

Link to this full text:

http://hdl.handle.net/2318/158704 


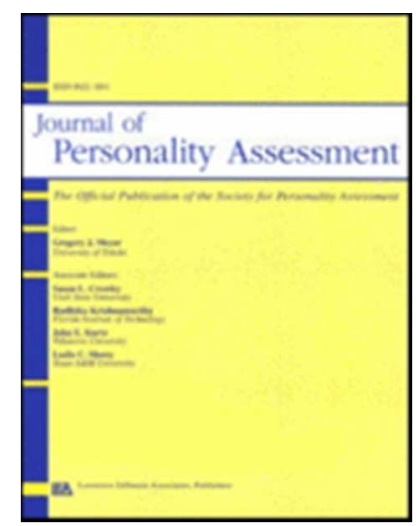

\section{Cross-Cultural Validation of the Rorschach Developmental Index}

\begin{tabular}{|r|l|}
\hline Journal: & Journal of Personality Assessment \\
\hline Manuscript ID: & JPA-2014-216.R1 \\
\hline Manuscript Type: & General Submission \\
\hline Keywords: & Developmental Index, Maturation, Rorschach < Measures, Validity \\
\hline \multicolumn{2}{|l}{} \\
\hline
\end{tabular}

\section{SCHOLARONE' \\ Manuscripts}


Cross-Validation of the DI

\begin{abstract}
The Developmental Index (DI) has recently been introduced as a composite Rorschach measure of psychological development and maturation, which can be used both with the Comprehensive System (Exner, 2003), and with the recently developed Rorschach Performance Assessment System (Meyer, Viglione, Mihura, Erard, \& Erdberg, 2011). As the DI is new, and its validity has not yet been investigated with independent non-U.S. samples, we tested the correlation between DI and age using three relatively large samples, two of which were from outside the U.S (total $\mathrm{N}=902$ ). Other Rorschach variables presumably associated with maturation, such as complexity and productivity, were also investigated. As expected, the DI significantly correlated with age, with small variations across the three samples. Importantly, the correlation between DI and age remained statistically significant also after controlling for productivity, i.e., the number of responses, and complexity. Keywords: Developmental Index; Maturation; Rorschach; Validity.
\end{abstract}




\section{Cross-Cultural Validation of the Rorschach Developmental Index}

It is well-known that, relative to adults, children and adolescents produce markedly different records when administered the Rorschach Inkblot Test (Rorschach, 1921). They typically produce less complex and less sophisticated records. Ames and colleagues (Ames, Metraux, \& Walker, 1971; Ames, Metraux, Rodell, \& Walker, 1974), for instance, reported notable age-based increases in variables such as number of responses $(\mathrm{R})$, whole locations (W), and human movement (M), and decreases in variables such as percentage of pure form responses (F\%), and percentage of common, easily seen detail responses (D\%). Similarly, the Comprehensive System (CS; Exner, 2003) normative reference samples show that the greater the age, the higher the mean scores of variables such as $M$, synthesis $(\mathrm{DQ}+)$, and form dominated color responses (FC) (Exner, 2003; Exner \& Weiner, 1994; Exner, Thomas, \& Mason, 1985; Weiner, 2003). Along the same line, a recent, internationally based Rorschach study encompassing 2,647 child and adolescent records from five countries (Meyer, Erdberg, \& Shaffer, 2007) also showed a similar developmental trend, with the Rorschachs becoming more complex and elaborated as the ages increase. In short, evidence from different contexts and perspectives indicates that as children and adolescents age and mature their Rorschach responses also change as their perceptions and verbalizations become more complex and articulated.

The process of maturation involves changes in cognitive, psychological, and social domains (Sigelman \& Rider, 2012). When compared to younger children, older individuals typically possess superior cognitive, social, and emotional abilities. Accordingly, as the age increases, children more competently manage the complex, demanding, and stressful challenges of everyday life. It is thought that this neurobiological maturation process continue until the mid-20s (Baltes, 1987; Thompson et al. 2000). To take into account the 
impact of such a developmental trend on psychological assessment, many psychological tools used with children and adolescents present different normative data for different age ranks (see, for example, Wechsler, 2003, 2008). Given that, it is not surprising that from early childhood to the mid-20s many Rorschach scores also vary with age.

To better understand the extent to which developmental progress and growth, as well as psychological maturation, can be observed and measured within the Rorschach Inkblot Test, Stanfill, Viglione, and Resende (2013) recently evaluated Rorschach protocols from a large data set comprised of individuals from different countries and examination contexts. The primary focus of their investigation was the relationship between age - used by the authors as a proxy marker of psychological development - and Rorschach variables. ${ }^{1}$ This effort culminated in the Developmental Index (DI), a composite Rorschach variable that can be used both with the CS, and with the recently developed Rorschach Performance Assessment System (R-PAS; Meyer, Viglione, Mihura, Erard, \& Erdberg, 2011).

The authors undertook a three-step process to create the DI: First, they evaluated the association with age (e.g., by examining Pearson correlations with age, child vs. adult comparisons, etc.) of 143 Rorschach variables, i.e., all the available CS scores and the Ego Impairment Index (EII-2; Perry, Viglione, \& Braff, 1992; Viglione, Perry, \& Meyer, 2003; for a newer version of the EII, see Viglione, Perry, Giromini, \& Meyer, 2011). This first step combined data from eleven published studies and one unpublished dataset, and resulted in the selection of 27 variables demonstrated to have the greatest association with age. The second step, in which the DI was created, evaluated an independent sample of 374 nonpatient Rorschach records, and utilized various regression-based methodologies to select the best combination of variables which, as a group, optimize the prediction of age. The final DI formula was generated in this second step. The variables in the final DI were: percentage of

\footnotetext{
${ }^{1}$ In some analyses, Stanfill et al. (2013) considered age in years; in others, they used Wechsler normative age brackets or ranges (Wechsler, 2003, 2008). Ultimately, however, their main purpose was "to produce an index that would map against age in years" (Stanfill et al., 2013, p. 180).
} 
pure form, inanimate object movement, reflection, texture, vista, form dimension, percentage of weak or distorted form quality, percentage of accurate or ordinary form quality, and anatomy, $\mathrm{x}$-ray, art, and sexual content. The DI is a weighted sum of these variables with coefficients derived from regression. In addition, each variable has a maximum allowable contribution to the DI (i.e., the score of each variable is truncated at $z= \pm 3$ ), so that any single variable does not dominate. The DI variables themselves confirm the association between age and accuracy (e.g., percentage of weak or distorted form quality [Xu\% and X$\%$, percentage of accurate or ordinary form quality [Xo\%]), productivity and complexity (e.g., percentage of pure form, shading-related determinants such as vista and texture), and sophistication in the response contents (e.g., art, x-ray).

In the final step, the DI was cross-validated with a large group which included children and adolescents from various clinical and forensic settings and also children with learning-disabilities $(N=277)$. The correlation between age and the DI was statistically significant, with a medium effect size $(r=.40)$. Importantly, such a result was achieved with a highly heterogeneous group of patients, offenders, and stressed individuals, "factors that obscure the expression of psychological development or to some degree are caused by immaturity" (Stanfill et al., 2013, p. 181). Thus, the authors speculated that with non-patient samples the relationship between age and the DI might be even stronger. Moreover, when the mean DI of this child and adolescent sample was contrasted to the mean DI of an adult sample $(n=233)$, a large effect size of Cohen's $d=1.15$ was observed, with the adults showing significantly higher scores. Of note, the DI predicted age group membership (children and adolescents vs. adults) more accurately than did Complexity (Dean, Viglione, Perry, \& Meyer, 2007; Meyer et al., 2011; Viglione, 1999), a Rorschach composite variable measuring differentiation and integration in the responses, as well as sophistication and task 
engagement. ${ }^{2}$ Indeed, examination of the receiver operating characteristic (ROC) curve for the DI and Complexity in predicting age group membership revealed that the area under curve (AUC) for the DI (.79) was notably larger than that for Complexity (.59). Based on these findings, Stanfill et al. (2013) speculated that as compared to Complexity, the DI might more accurately assess and identify age-based changes presumably associated with psychological maturation. Of course, given the limited information currently available, more research on this claim would certainly be beneficial.

According to Stanfill et al. (2013), the main interpretative function of the DI would be to allow comparisons between the client's level of psychological development and his or her chronological age. Said differently, the DI aims at identifying immaturity and precociousness in applied, clinical work. Albeit with large individual differences, indeed, age may be used "to specify average developmental status or expected changes across a variety of domains in functioning” (Stanfill et al., 2013, p. 174). Thus, by comparing an individual's DI against his or her age-based, expected value, it might be possible to gain information regarding the respondent's level of psychological maturation. It should be pointed out, however, that to date no studies have yet replicated the findings reported by Stanfill et al. (2013), and the exact extent to which the DI might in fact associate with immaturity or psychopathology is currently unknown. Therefore, additional research on the DI is needed.

\begin{abstract}
Aim of the Study
As the DI is new and its validity has not yet been investigated with independent nonU.S. samples, we aimed at studying the relationships between the DI, age, Complexity, and $\mathrm{R}$, using three relatively large samples. More specifically, we tested the correlation between
\end{abstract}

\footnotetext{
${ }^{2}$ Complexity is an aggregate measure of the total amount of differentiation and integration in a Rorschach record. It encompasses three components: (1) information regarding the location, the use of the space, and the quality of response objects; (2) number and complexity of response contents; (3) number and complexity of response determinants. For additional information, see Meyer et al. (2011) and Viglione (1999).
} 
the DI and age within two Italian samples and one U.S. sample, and examined the extent to which the DI correlates with age after controlling for Complexity and R.

\section{Method}

Because biological and cognitive maturation are largely complete by about age of 25 (Baltes, 1987; Thompson et al., 2000), we decided to use age $\leq 25$ as inclusion criteria for this child, adolescent, and young adult study. This was the same cutoff used in original DI research for calculating correlations and regression with age. No other inclusion or exclusion criteria were set. The resulting, combined data set consisted of 902 Rorschach records from three independent clinical samples. All were retrieved electronically from a number of clinical, de-identified, computerized databases to which the authors had access. The relationships between the DI, age, Complexity, and R were then examined, both within each sample, and within the entire, combined sample. These variables were selected as foils since they are associated with complexity, productivity, and presumably maturation (Exner, 2003; Meyer et al, 2011; Stanfill et al, 2013; Vigione, 1999).

\section{Participants}

The final, combined sample included 902 children, adolescents, and young adults ranging in age from 5 to 25 (mean age $=16.3, \mathrm{SD}=4.9$ ). Of these 902 records, 793 were collected and coded using the standard, CS guidelines (Exner, 2003), while 109 were collected using a nearly final version of the R-PAS administration (Meyer et al., 2011), but coded with the CS. About half were boys (50.7\%), and half girls (49.3\%). About 70\% were administered in Italy, specifically, 562 in Milan and 68 in Turin, with the remaining 272 being administered in Ohio, in the U.S. Additional demographic information is detailed below and in Table 1. All Rorschachs were administered by expert clinicians holding graduate degrees in psychology or pre-doctoral psychology interns, all trained in CS techniques. 
The differences in age between the Milan, Turin, and Ohio samples were statistically significant, $F(2,899)=124.3, p<.01$, with the Milan sample being the oldest sample $(M=$ $17.9, S D=4.9)$, and the Ohio sample the youngest one $(M=12.9, S D=3.3)$ (all post hoc comparisons significant after Bonferroni correction). Furthermore, these three samples were also significantly different in terms of gender distribution, $X^{2}(2)=21.0, p<.01$, with the Ohio sample including relatively more boys (i.e., $60 \%)$ compared to the Milan (50\% of boys) and Turin ( $45 \%$ of boys) samples, as demonstrated by examination of the standardized residuals. $^{3}$ Although the levels of education and diagnostic composition of the samples were not available for all the data, it is highly likely that the three samples also differed with respect to these variables. As such, one should keep in mind that the entire, combined sample is in fact highly heterogeneous.

The Milan Sample. The Milan sample included 562 archival Rorschach protocols, extracted from computerized records of child, adolescent, and young adult outpatients who were referred for psychological help at a private clinic in Milan. All Rorschachs were administered following the standard CS guidelines, as part of a routine assessment procedure. All examiners were expert clinicians who had received intensive training in CS administration, coding, and interpretation, and had used the Rorschach for many years. Although diagnoses and socioeconomic status were not well documented, the clinic from which the database was obtained is known to serve predominantly clients with middle-high socioeconomic means, and who seek help for depression and/or anxiety. Diagnoses are usually made by licensed clinicians with several years of experience. Ages ranged from 5 to 25 , with a mean age of $17.9(\mathrm{SD}=4.9)$. The general distribution was roughly equal $(45.1 \%$ were boys, $54.9 \%$ were girls). Education and ethnicity were not documented, but the majority of the sample was known to be Italian and Caucasian, and all spoke Italian.

\footnotetext{
${ }^{3}$ Within the Ohio sample, there are no significant differences between the CS and early R-PAS administration groups with regards to age and gender.
} 
The Turin Sample. The Turin sample consisted of 68 archival Rorschach protocols, extracted from computerized records of children, adolescents, and young adults hospitalized, by order of juvenile court, in therapeutic communities in Piedmont, a region of northern Italy. All Rorschachs were administered according to the standard CS guidelines, as part of a routine standard procedure. All examiners were expert clinicians who had been trained in CS administration, coding, and interpretation for many years. Although diagnoses and socioeconomic status were not well documented, it was known that most of the sample had a severe Personality Disorder (PD), mainly Borderline, Paranoid or Antisocial, and had lower socioeconomic means. Diagnostic evaluations were typically conducted by expert clinicians who worked in the field for several years. Ages ranged from 11 to 23, with a mean age of 16.1 $(\mathrm{SD}=2.3)$. Half $(n=34)$ were boys, and half $(n=34)$ girls. Education ranged from 3 to 14 years, with a mean of $8.7(\mathrm{SD}=1.8)$. Ethnicity was not documented, but all clients spoke Italian.

The Ohio Sample. The Ohio sample consisted of 272 archival Rorschach protocols, extracted from computerized records of children, adolescents, and young adults who referred for psychological help at an outpatient community mental health agency based in Canton, Ohio. More than half of these records, i.e., 163, were collected using the standard CS guidelines. The remaining 109 were instead collected using a nearly finalized version of the recently developed R-PAS, but coded with the CS. Part of these data were discussed in Reese, Viglione, and Giromini (2014). In terms of diagnostic description, 25.7\% had a mood disorder, $16.9 \%$ had a primary diagnosis of $\mathrm{ADHD}, 16.9 \%$ were court-involved due to sexually inappropriate behavior, $16.5 \%$ had PTSD, 11.4\% had an anxiety disorder (excluding PTSD), 7.0\% had a primary behavior disorder diagnosis (excluding ADHD), $4.0 \%$ had an Autism Spectrum Disorder, and 1.5\% had a psychotic disorder. The socioeconomic status was not well documented; however, the agency from which the database was obtained is 
known typically to serve clients with lower socioeconomic means. Ages ranged from 5 to 20, with a mean age of $12.9(\mathrm{SD}=3.3)$. About two-thirds $(62.1 \%)$ were boys, and one third $(37.9 \%)$ girls. Education ranged from 0 to 12 years, with a mean of $7.4(\mathrm{SD}=3.2)$ years. With regard to racial identity, 76.5\% Caucasian, 14.3\% African American, 1.1\% Hispanic, $.4 \%$ Asian American, 7.7\% Other (Multiracial). All were English speaking.

\section{Inter-rater Reliability}

To establish inter-rater reliability, a total of ninety records (45 from the Milan sample, 25 from the Turin sample, and 20 from the Ohio sample) were randomly selected and coded by a number of independent raters, who were blind to the original codes. As for the Milan and Turin samples, the second, independent rater was an Italian doctoral student who had about ten years of clinical and research experience using the CS, and had previously coded more than 100 Rorschachs. As for the Ohio sample, ten records had formerly been independently coded by both the examiner and his or her supervisor, and ten records were recoded by an American, advanced graduate student who had previously coded more than 100 Rorschach records ${ }^{4}$. Two-way random effects model single measures intraclass correlation coefficients (ICCs) were calculated so as to establish the inter-rater reliability of the three variables under investigation, i.e., the DI, Complexity, and $\mathrm{R}^{5}$. Results reported in Table 2 indicate that all the measures included in this study had excellent inter-rater reliability (for ICC benchmarks interpretation, see Cicchetti, 1994, and Shrout \& Fliess, 1979).

\section{Data Analysis}

To investigate the relationships between the DI, age, Complexity, and R, we separately analyzed each of the three samples, as well as the entire, combined sample. First,

\footnotetext{
${ }^{4}$ Additional information about the inter-rater reliability of the Ohio sample is detailed in Reese et al. (2014). Reese and colleagues, however, did not report data on the inter-rater reliability of the DI.

${ }^{5}$ It should be noted that a rater does not directly compute the DI or Complexity. These composite variables are calculated based on relatively complex equation formulas, which weight the scores of other Rorschach variables. Thus, each entire protocol was first rescored, and the DI and Complexity were then computed 'a posteriori' to calculate inter-rater reliability.
} 
we tested the Pearson bivariate correlations between age and the three Rorschach variables under investigation, i.e., the DI, Complexity, and R. Then, we performed two partial correlations aimed at investigating the extent to which the DI correlated with age after removing, one at a time, Complexity and $\mathrm{R}$.

\section{Results}

The mean values of the DI, Complexity, and R are reported in Table 3. In all cases, the CS and R-PAS Ohio sub-samples produced virtually identical scores, with no statistically significant differences. Conversely, when compared to each other, the Milan, Turin, and Ohio samples significantly differed for all of the variables under investigation, $F(2,899) \geq 4.76$, all $p<.01{ }^{6}$ Post hoc comparisons, in particular, showed that: (1) the Milan sample produced a significantly higher mean DI value than both the Turin $(d=.62)$ and Ohio samples $(d=.59)$; (2) the Milan sample produced a significantly higher Complexity value than the Turin sample $(d=.30)$; (3) the Turin sample produced a significantly lower number of responses (R) than both the Milan $(d=.33)$ and Ohio $(d=.53)$ samples. None of the other various post hoc comparisons reached statistical significance. ${ }^{7}$ Thus, the Milan sample - which is the sample with the highest mean age, among the three under investigation - produced the highest DI and Complexity values.

We then further investigated the association between the DI and age. Similar to Stanfill et al.'s (2013) findings, the DI correlated significantly with age, with $r$ ranging from $.28(p<.01)$ to $.41(p<.01)$, depending on the sample under consideration (Table 4$)$.

Complexity also correlated with age. In contrast, $\mathrm{R}$ did not significantly correlate with age.

Critically, in all cases the DI produced the highest correlational values.

Finally, a series of partial correlations were also tested. As reported in Table 5, the DI continued to be associated with age when the effects of Complexity and $\mathrm{R}$ were partialled

\footnotetext{
${ }^{6}$ These results refer to analyses conducted after collapsing the two Ohio sub-samples. Similar conclusions, however, can be taken also when these two sub-samples are not combined.

${ }^{7}$ For all comparisons listed here, alpha was adjusted using Bonferroni correction.
} 
out. Conversely, when the effect of the DI was partialled from the correlations between age and Complexity and $\mathrm{R}$, none of the correlations were significant.

\section{Discussion}

To provide independent and cross-cultural validity information about the recently introduced Developmental Index (DI; Stanfill et al., 2013), we retrieved archival data for a total of 902 child, adolescent, and young adult Rorschach records, and investigated the relationships between the DI, age, and other Rorschach variables presumably associated with progress and growth. As expected, the DI significantly correlated with age, with small variations across the three samples from two countries.

As reported by Stanfill et al. (2013) in their DI development paper, the correlation between the DI and age, calculated with an independent, clinical group of 277 children, was medium and significant, with $r=.40$. In our study, the correlation between the DI and age ranged between .28 to .38 when the individual, specific samples were analyzed, and reached .42 when entire, combined samples were taken into consideration. Thus, virtually no shrinkage occurred from Stanfill et al.'s (2013) study. Accordingly, since large samples, from quite different examination and cultural contexts, have at this point been investigated, one may anticipate these correlation values to remain stable and be observed also in future studies across cultures and geography.

As reviewed in the Introduction, prior to Stanfill et al.'s (2013) work, the best Rorschach markers of psychological development, progress, and growth were variables presumably associated with productivity, complexity and sophistication (Meyer et al., 2011; Viglione, 1999). Based on this, we tested a series of partial correlations to investigate whether the DI would remain correlated with age also after controlling for R and Complexity. In line with Stanfill et al.'s (2013) findings, these analyses confirmed that the DI has a unique association with age. Conversely, neither R nor Complexity remained correlated with age 
when we controlled for the DI. Thus, our study provides additional support for the incremental validity of the DI, compared to Complexity and R, as a measure of age-based changes in youth. Moreover, if one considers age as a proxy for growth and maturation, the DI has potential as a marker of psychological development. Of course, though, future research with developmental criteria, beyond age, is needed, to test whether the DI actually measures development and, once accomplished, to specify the nature of that relationship.

It is noteworthy that $\mathrm{R}$ did not correlate with age. On the one hand, this finding was unexpected, based on some past research conducted by Ames and colleagues (Ames et al., 1971; Ames et al., 1974). On the other hand, however, a lack of relationship between age and R was also reported in a large, international study by Meyer et al. (2007). Further research on this topic is thus recommended.

The effect size of the correlation in our sample between DI and age reveals that most of the variance of age is not accounted for by the DI within our sample. Future studies might contrast clinical samples to non-patient samples to investigate the hypotheses that emotional and behavioral problems are associated with atypical developmental paths. If true, one would expect that the both correlation between the DI and maturity and the DI mean itself to be lower in patient and problematic samples like those in our study.

The most obvious limitation of the current study is its lack of precise demographic and diagnostic information. Additional controlled research with better characterization of these variables is therefore needed, to investigate the extent to which the DI may be affected by gender, culture and/or psychopathology. Nevertheless, the current study still provides valuable information, from large and culturally diverse samples, strongly supporting the cross-cultural validity of the recently developed DI. 
Cross-Validation of the DI

\section{References}

Ames, L. B., Metraux, R. W., \& Walker, R. B. (1971). Adolescent Rorschach responses: Developmental trends from ten to sixteen years. New York, NY: Brunner/Mazel.

Ames, L. B., Metraux, R. W., Rodell, J. L., \& Walker, R. B. (1974). Child Rorschach responses: Developmental trends from two to ten years. New York, NY: Brunner/Mazel.

Baltes, P. B. (1987). Theoretical propositions of life-span developmental psychology: On the dynamics between growth and decline. Developmental Psychology, 23, 611-626.

Brainard, R. (2004). A comparison of learning disabled children and nonlearning disabled children on the Rorschach: An information processing perspective (Unpublished doctoral dissertation). San Diego, CA: California School of Professional Psychology.

Cheyette, L. (1997). The effects of examiner style on Rorschach test results of children with borderline psychopathology (Unpublished doctoral dissertation). San Diego, CA: California School of Professional Psychology.

Cicchetti, D. V. (1994). Guidelines, criteria, and rules of thumb for evaluating normed and standardized assessment instruments in psychology. Psychological Assessment, 6, 284-290.

Dean, K. L., Viglione, D. J., Perry, W., \& Meyer, G. J. (2007). A method to optimize the response range while maintaining Rorschach comprehensive system validity. Journal of Personality Assessment, 89, 149-161.

Delucas, A. L. (1997). The quality of paternal attachment and violent behavior: A Rorschach study of convicted military male offenders (Unpublished doctoral dissertation). San Diego, CA: California School of Professional Psychology.

Exner, J. E., Jr. (2003). The Rorschach: A comprehensive system: Vol. 1. Basic foundations and principles of interpretation (4th ed.). New York, NY:Wiley. 
Exner, J. E., Jr., \& Weiner, I.B. (1994). The Rorschach: A comprehensive system: Vol. 3. Assessment of children and adolescents. New York, NY: Wiley.

Exner, J. E., Jr., Thomas, E. A., \& Mason, B. (1985). Children’s Rorschachs: Description and prediction. Journal of Personality Assessment, 49(1), 13-20.

Jansak, D. (1996). The Rorschach Comprehensive System Depression Index, depression heterogeneity, and the role of self-schema (Unpublished doctoral dissertation). San Diego, CA: California School of Professional Psychology.

Kates, J. (2004). A validation study of the Rorschach Ego Impairment Index (Unpublished doctoral dissertation). San Diego, CA: California School of Professional Psychology.

Meyer, G. J., Erdberg, P., \& Shaffer, T. W. (2007). Toward international normative reference data for the Comprehensive System. Journal of Personality Assessment, 89(S1), S201-S216.

Meyer, G. J., Viglione, D. J., Mihura, J. L., Erard, R. E., \& Erdberg, P. (2011). Rorschach Performance Assessment System: Administration, coding, interpretation, and technical manual. Toledo, OH: Rorschach Performance Assessment System.

Perry, W., Viglione, D. J., \& Braff, D. (1992). The Ego Impairment Index and schizophrenia: A validation study. Journal of Personality Assessment, 59(1), 165-175.

Reese, J. B., Viglione, D. J., \& Giromini, L. (2014). A Comparison Between Comprehensive System and an Early Version of the Rorschach Performance Assessment System Administration With Outpatient Children and Adolescents. Journal of Personality Assessment, DOI: 10.1080/00223891.2014.889700

Rorschach, H. (1921). Psychodiagnostik [Psychodiagnostics]. Bern, Switzerland: Bircher. Shrout, P. E., \& Fliess, J. L. (1979). Intraclass correlations: Uses in assessing rater reliability. Psychological Bulletin, 86, 420-428. 
Sigelman, C. K., \& Rider, E. A. (2012). Life-span human development, (7th ed.). Belmont, CA: Wadsworth.

Stanfill, M. L. (2010). Examination of a juvenile sex offender typology as measured by the Rorschach Inkblot Test, cognitive functioning, and victim and offense characteristics (Unpublished doctoral dissertation). San Diego, CA: California School of Professional Psychology.

Stanfill, M. L., Viglione, D. J., \& Resende, A. C. (2013). Measuring psychological development with the Rorschach. Journal of Personality Assessment, 95(2), 174-186.

Strauss, K. (1996). Differential diagnosis of battered women through psychological testing: Personality disorder or post traumatic stress disorder? (Unpublished doctoral dissertation). San Diego, CA: California School of Professional Psychology.

Thompson, P. M., Giedd, J. N., Woods, R. P., MacDonald, D., Evans, A. C., \& Toga, A. W. (2000). Growth patterns in the developing brain detected by using continuum mechanical tensor maps. Nature, 404(6774), 190-193.

Viglione, D. J. (1999). A review of recent research addressing the utility of the Rorschach. Psychological Assessment, 11, 251-265.

Viglione, D. J., Perry, W., Giromini, L., \& Meyer, G. J. (2011). Revising the Rorschach Ego Impairment Index to accommodate recent recommendations about improving Rorschach validity. International Journal of Testing, 11, 349-364.

Viglione, D. J., Perry, W., \& Meyer, G. J. (2003). Refinements in the Rorschach Ego Impairment Index incorporating the Human Representational variable. Journal of Personality Assessment, 81, 149-156.

Wechsler, D. (2003). Wechsler Intelligence Scale for Children-Fourth edition: Technical and interpretive manual. San Antonio, TX: Psychological Corporation. 
Wechsler, D. (2008). Wechsler Adult Intelligence Scale-Fourth edition: Technical and interpretive manual. San Antonio, TX: Pearson.

Weiner, I. B. (2003). Principles of Rorschach interpretation. New York, NY: Routledge/Taylor \& Francis. 
Cross-Validation of the DI

Table 1. Composition of the Sample

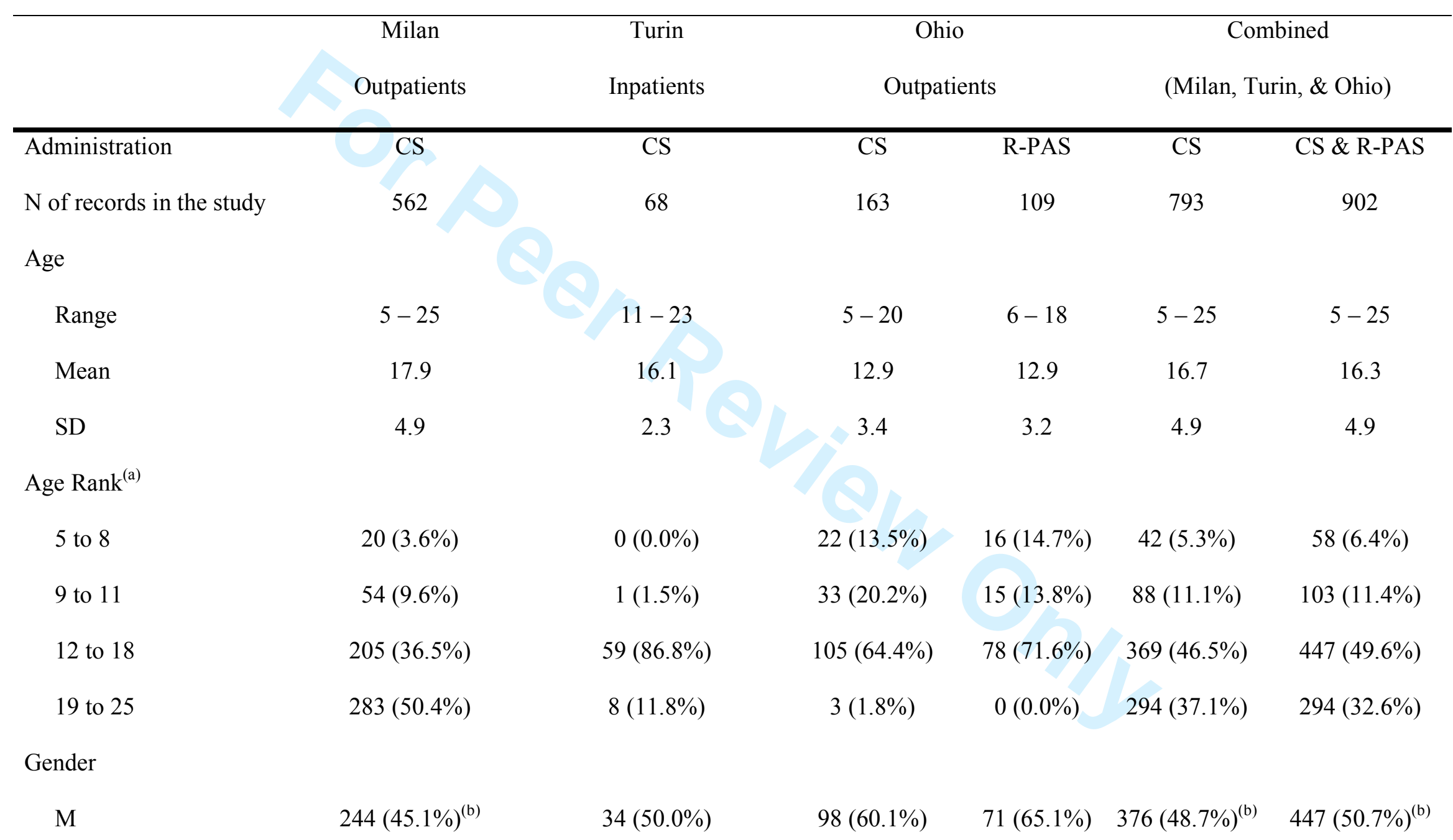


Cross-Validation of the DI

F

$297(54.9 \%)^{(b)} \quad 34(50.0 \%)$

$65(39.9 \%)$

$38(34.9 \%) \quad 396(51.3 \%)^{(b)}$

$434(49.3 \%)^{(b)}$

Education

Range

Not available

$3-14^{\text {(c) }}$

$0-12$

$1-12$

$0-14^{(\mathrm{d})}$

$0-14^{(\mathrm{d})}$

Mean

Not available

$8.7^{(\mathrm{c})}$

7.3

7.5

$7.7^{(\mathrm{d})}$

$7.6^{(\mathrm{d})}$

SD

Not available

$1.8^{(\mathrm{c})}$

3.2

3.1

$3.0^{(\mathrm{d})}$

$3.0^{(\mathrm{d})}$

a - age ranks refer to age groupings used to generate the normative data for the Wechsler intelligence tests (Wechsler, 2003, 2008); b - these are

valid percentages, 21 records were missing information on gender; $\mathrm{c}$ - three records were missing information on education; $\mathrm{d}-565$ records were missing information on education. 
1

2

8

9

11
12

13

14

15

16

17

18

19

20

21

22

23

24

25

26

27

28

29

30

31

32

34

35

36

37

38

39

40

41

42

43

44

45

46

47

48

URL: http://mc.manuscriptcentral.com/JPersAssess Email: jpa_office@emich.edu

Cross-Validation of the DI

Table 2. Inter-rater Reliability

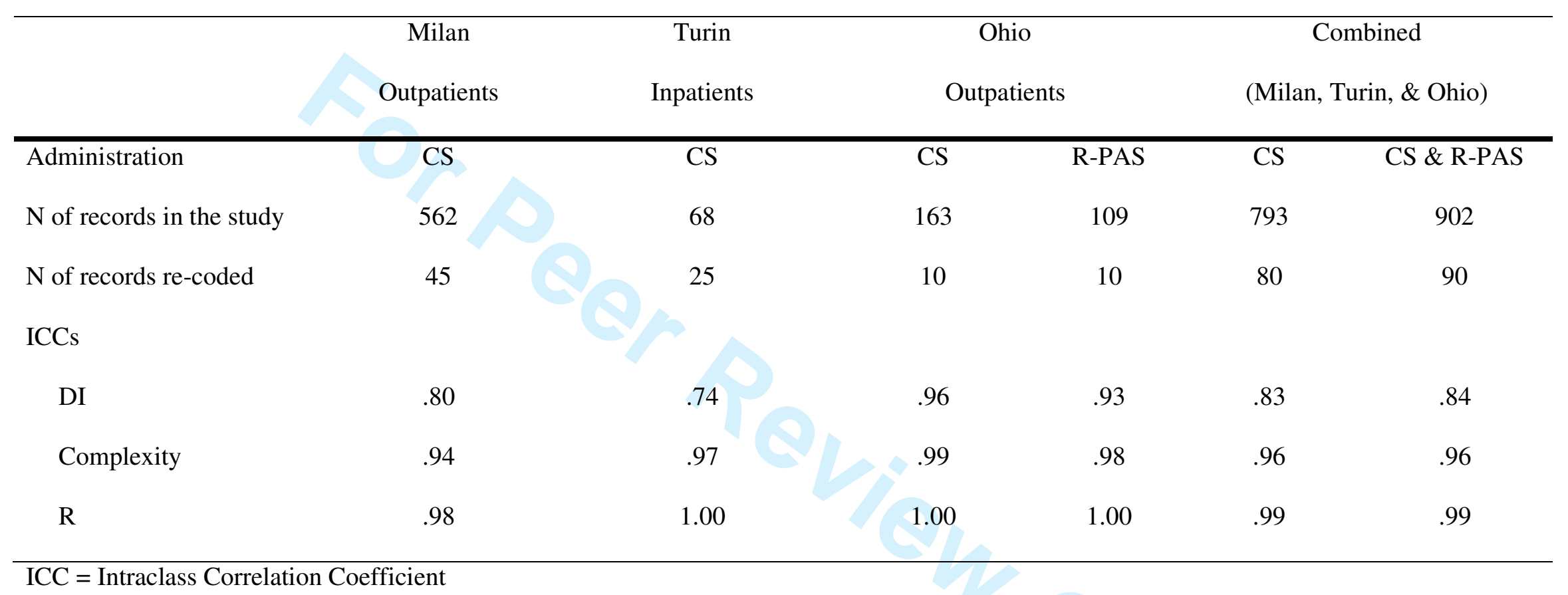


Cross-Validation of the DI

Table 3. Descriptive Statistics.

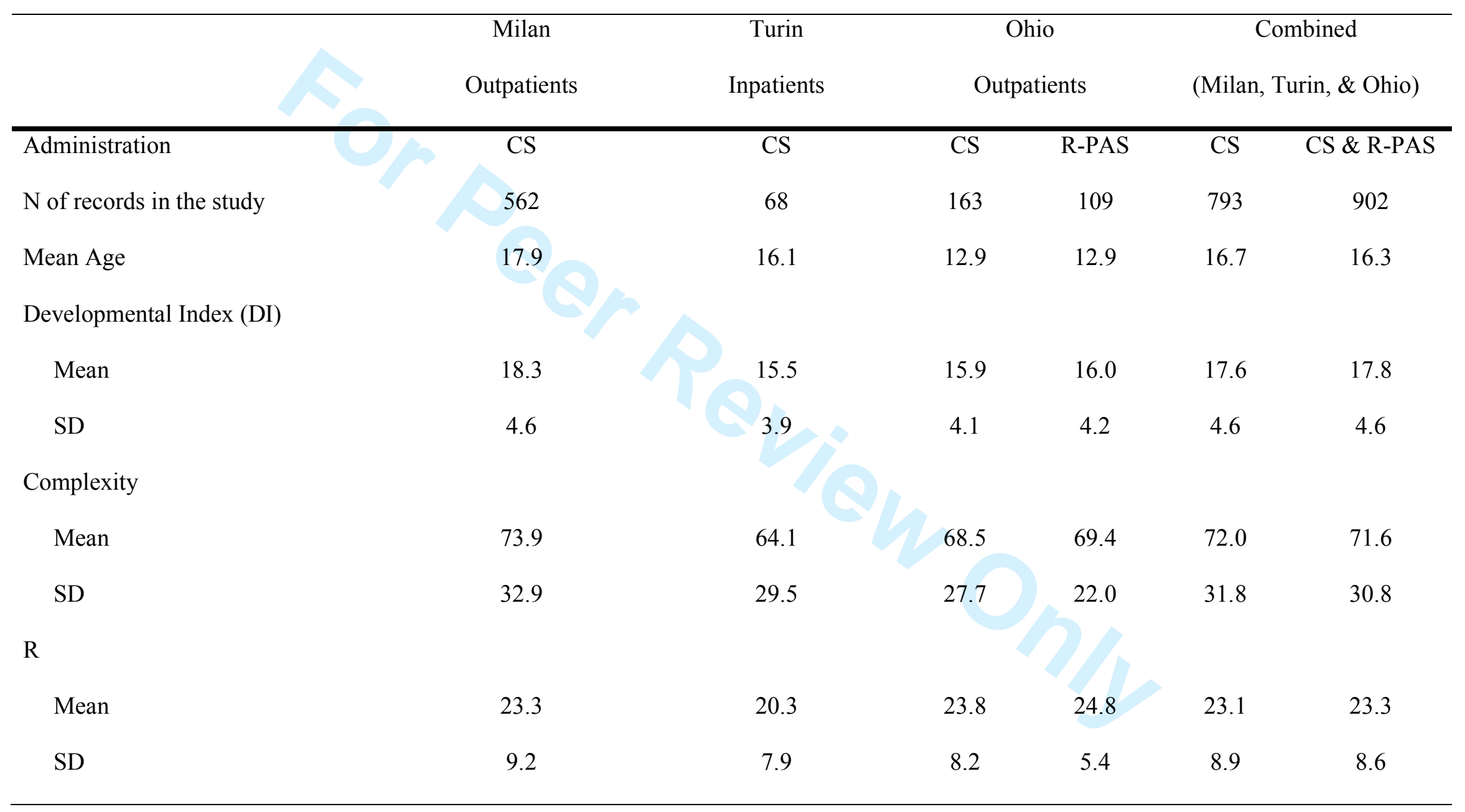


Cross-Validation of the DI

Table 4. Correlations between Age, the DI, Complexity, and R.

\begin{tabular}{|c|c|c|c|}
\hline & Age & DI & Complexity \\
\hline \multicolumn{4}{|c|}{ Milan Sample - CS $(\mathrm{N}=562)$} \\
\hline DI & $.36^{* *}$ & & \\
\hline Complexity & $.15^{* *}$ & $.43 * *$ & \\
\hline $\mathrm{R}$ & .07 & $.21 * *$ & $.81 * *$ \\
\hline \multicolumn{4}{|c|}{ Turin Sample - CS $(\mathrm{N}=68)$} \\
\hline DI & $.34 * *$ & & \\
\hline Complexity & .23 & $.49 * *$ & \\
\hline $\mathrm{R}$ & .02 & .18 & $.73 * *$ \\
\hline \multicolumn{4}{|c|}{ Ohio Sample - CS $(\mathrm{N}=163)$} \\
\hline DI & $.28 * *$ & & \\
\hline Complexity & .04 & $.40 * *$ & \\
\hline $\mathrm{R}$ & .02 & $.17^{*}$ & $.73 * *$ \\
\hline \multicolumn{4}{|c|}{ Ohio Sample - R-PAS (N = 109) } \\
\hline DI & $.38 * *$ & & \\
\hline Complexity & .07 & $.42 * *$ & \\
\hline $\mathrm{R}$ & -.01 & .12 & $.61 * *$ \\
\hline
\end{tabular}

Combined $-\mathrm{CS}(\mathrm{N}=793)$

DI $.39 * *$
Complexity $.16^{* *} \quad .44^{* *}$
$\begin{array}{llll}\mathrm{R} & .05 & .20 * * & .79 * *\end{array}$

Combined - CS \& R-PAS $(\mathrm{N}=902)$

10
DI

Complexity

\section{$.41 * *$}

$.15^{* *} \quad .44^{* *}$ 


\section{Cross-Validation of the DI}

2 


\section{Cross-Validation of the DI}

Table 5. Partial Correlations between Age and the DI, by Complexity and R.

\begin{tabular}{|c|c|c|}
\hline & By Complexity & By R \\
\hline \multicolumn{3}{|c|}{ Milan Sample $-\mathrm{CS}(\mathrm{N}=562)$} \\
\hline DI and Age & $.33 * *$ & $.35 * *$ \\
\hline \multicolumn{3}{|c|}{ Turin Sample - CS $(\mathrm{N}=68)$} \\
\hline DI and Age & $.27 *$ & $.35 * *$ \\
\hline \multicolumn{3}{|c|}{ Ohio Sample - CS $(\mathrm{N}=163)$} \\
\hline DI and Age & $.29 * *$ & $.28 * *$ \\
\hline \multicolumn{3}{|c|}{ Ohio Sample - R-PAS (N = 109) } \\
\hline DI and Age & $.39 * *$ & $.39 * *$ \\
\hline \multicolumn{3}{|c|}{ Combined - CS $(\mathrm{N}=793)$} \\
\hline DI and Age & $.37 * *$ & $.39 * *$ \\
\hline \multicolumn{3}{|c|}{ Combined - CS \& R-PAS $(\mathrm{N}=902)$} \\
\hline DI and Age & $.39 * *$ & $.42 * *$ \\
\hline
\end{tabular}

